\title{
Review of Recent Progress of Perovskite Solar Cells Without Spiro-OMeTAD
}

\author{
Vijaya Shankar, Teresa Demeritte and Qilin Dai* \\ Department of Physics, Atmospheric Sciences and Geoscience, Jackson State University, Jackson, Mississippi, USA
}

\begin{abstract}
Received: September 28, 2016; Accepted: September 29, 2016; Published: October 04, 2016
*Corresponding author: Department of Physics, Atmospheric Sciences and Geoscience, Jackson State University, Jackson, Mississippi 39217, USA, E-mail: qilin.dai@jsums.edu
\end{abstract}

\begin{abstract}
The Power Conversion Efficiency (PCE) of Perovskite Solar Cells (PSCs) has been boosted to over 20\%. However, it is still challenging to extend the PSCs to outdoor applications, such as the cost and the stability of the devices. The reported PSCs with high PCE are all based on Spiro-OMeTAD, which is a very expensive material. SpiroOMeTAD as whole transporting material (HTM) protects perovskite material from degradation. The poor stability of the PSCs also can be improved by a better HTM. Some researchers are investigating new type of HTMs with an emphasis on excellent charge transfer efficiency and high stability. This review summarizes recent studies of the progress of PSC device performance with new type of HTM, instead of Spiro-OMeTAD.
\end{abstract}

\section{Introduction}

PSCs have been studied extensively recently due to their high record (over 20\%) within four years due to the excellent photoelectrical properties of perovskite material [1]. The high PCE may meet the requirement of outdoor applications, which is comparable to silicon solar cells. However, it is still challenging to utilize PSCs for practical application based on the current research due to their poor stability and cost issues. The poor stability is caused by the perovskite material sensitive to the moisture and the high cost is due to the Spiro-OMeTAD and other materials. All the reports about high PCE of PSCs are based on Spiro-OMeTAD as HTM, and it is reported that the protection of Spiro-OMeTAD to perovskite material is not very well due to the pin-holes in Spiro-OMeTAD layer leading to the moisture penetration into the devices and degradation of the devices. The preparation of Spiro-OMeTAD layer is reported to be spin coating which is related with the uniform film and unavoidable pin-holes [2]. The deposition method with the ability of preparing uniform thin films is not suitable to be applied to Spiro-OMeTAD due to the low decomposition temperature of the organic material. Therefore, the Spiro-OMeTAD is associated with stability and the cost of PSCs, and the investigation of other HTM is very critical to the development of PSCs. Some researchers explore some new type of PSCs without Spiro-OMeTAD. In this mini review, we summary the recent progress about the PSCs without SpiroOMeTAD including the PCE, stability and the device engineering.

\section{Discussion}

[5] Reported a PSC structure without a whole conductor in their devices, and a PCE of $12.8 \%$ was obtained in their study [3]. The perovskite solar cell is constructed by using the mesoporous layers of $\mathrm{TiO}_{2}$ and $\mathrm{ZrO}_{2}$ which are deposited on FTO covered glass substrate, and the cell structure does not require a hole conducting layer. The stability of this type of PSCs is more than $1000 \mathrm{~h}$ in ambient air under full sunlight. This is the first report about the PSC stability under full sunlight. The big achievement is attributed to the $\mathrm{ZrO}_{2}$ since the devices without $\mathrm{ZrO}_{2}$ structure were also investigated in their work. $\mathrm{ZrO}_{2}$ can block the flow of photo generated electrons to the back contact preventing recombination with the holes from the perovskite at the back contact leading to high PCE. Figure 2B shows another type of PSCs without Spiro-OMeTAD. In this structure, PEDOT: PSS was used as HTM; PCBM extracts electrons from perovskite solar cells. This structure is derived from organic solar cells, which usually have poor stability. However, recently [4] reported a PCE of $12.52 \%$ with this structure and optimized the stability by two-dimensional ruddles den-popper structure (Figure 2B) [4]. The stability was increased to $2250 \mathrm{~h}$ under constant, standard illumination. [5] Tried several of HTMs by the traditional PSC structure, where $\mathrm{TiO}_{2}$ was used as electron transport layer [5]. Table 1C shows the photovoltaic performance of PSCs based on different HTMs [5]. It can be seen that the efficiency of TTF based devices is compared with that of Spiro-OMeTAD, while P3HT based PSCs is not as good as Spiro-OMeTAD. The efficiency of TTF based PSCs decreased to $9 \%$ from 10\% under ambient atmosphere, compared to $1 \%$ for Spiro-OMeTAD based devices.

\section{Conclusion}

We reviewed the research progress of PSCs without SpiroOMeTAD. The stability is much better than that of Spiro-OMeTAD 

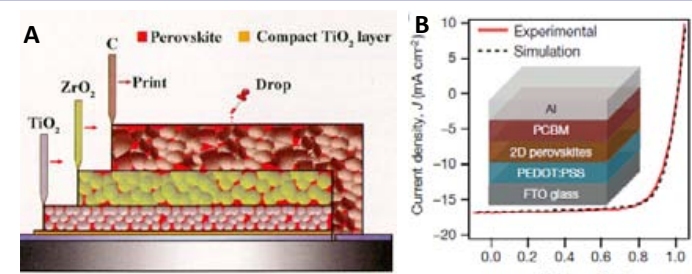

C

$$
J_{\mathrm{SC}}
$$

\begin{tabular}{llllrr} 
HTM (form) & $\begin{array}{l}J_{\mathrm{SC}} \\
\left(\mathrm{mA} \mathrm{cm}^{-2}\right)\end{array}$ & $V_{\mathrm{OC}}(\mathrm{v})$ & $\mathrm{FF}$ & $\eta(\%)$ & $R_{\mathrm{s}}(\Omega)$ \\
\hline TTF-1 (pristine) & 19.9 & 0.86 & 0.644 & 11.03 & 64.3 \\
TTF-1 (doped) & 12.1 & 0.83 & 0.439 & 4.41 & 162.4 \\
P3HT (pristine) & 12.8 & 0.80 & 0.607 & 6.22 & 72.1 \\
P3HT (doped) & 13.0 & 0.81 & 0.638 & 6.72 & 67.2 \\
Spiro-OMeTAD (pristine) & 15.1 & 0.88 & 0.465 & 6.18 & 158.2 \\
Spiro-OMeTAD (doped) & 19.8 & 0.91 & 0.631 & 11.4 & 70.6
\end{tabular}

Figure 1: A Schematic drawing of a hole-conductor-free PSC. From [A. Mei, X. Li, L. Liu, Z. ku, T. Liu, Y. Rong, M. Xu, M. Hu, J. Chen, Y. Yang, M. Gratzel and H. Han, Science.2014, 345,295] Reprinted with permission from AAAS. B another choice of the PSC without Spiro-OMeTAD and the corresponding J-V curves for the planar devices without From [H. Tsai, W. Nie, J. C. Blancon, C. C. Stoumpos, R. Asadpour, B. Harutyunyan, A. J. Neukirch, R. Verduzco, J. J. Crochet, S. Tretiak, L. Pedesseau, J. Eren, M. A. Alam, G. Gupta, J. Lou, P. M. Ajayan, M. J. Bedzyk, M. G. Kanatzidis and A.D. Mohite. Nature 2016.313-316.] Reprinted with permission from Nature Publishing Group under license number 3954941219208.C photovoltaic performance of the PSCs based on different HTM. From [J.Liu, Y.Wu, C.Qin, X.Yang, T.Yasudha, A.Islam, K.Zhang, W.Peng, W.Chen and L.Han. 2014. Energy.Environ. Sci, 7,2963-2967.] Reprinted with permission from Royal Society of Chemistry under license number 3957661474912. but the efficiency of the current results is not that high as SpieoOMETAD. Therefore, we think more research effort will be focused on improving the PCE and keep the current long term stability.

\section{Acknowledgements}

This work was supported by National Science Foundation under award \#1332444.

\section{References}

1. Teddy Salim, Shuangyong Sun, Yuichiro Abe, Anurag Krishna, Andrew C.Grimsdalea and Yeng Ming Lam. Perovskite-based solar cells: impact of morphology and device architecture on device performance. J. Mater. Chem. A. 2014;40:44.

2. Cong Chen, Yu Cheng, Qilin Dai and Hongwei Song. Radio Frequency Magnetron Sputtering Deposition of TiO2 Thin Films and Their Perovskite Solar Cell Applications. Sci.Rep. 2015;5:17684.

3. Mei A, Li X, Liu L, Ku Z, Liu T, Rong Y, et al. A hole-conductor-free, fully printable mesoscopic perovskite solar cell with high stability. Science. 2014;345(6194):295-298. doi:10.1126/science.1254763.

4. HT sai, W Nie, JC Blancon, CC Stoumpos, R Asadpour, AD Mohite, et al. High-efficiancy two dimensional Ruddlesden popper perovskite solar cells. Nature. 2016:313-316. doi:10.1038/nature18306.

5. J Liu, Y Wu, C Qin, X Yang, T Yasudha, A Islam, et al. A dopant-free holetransporting material for efficient and stable perovskite solar cells. Energy.Environ. Sci. 2014;7:2963-2967. DOI: 10.1039/C4EE01589D. 